\title{
ATM-Mediated Phosphorylation of Cortactin Involved in Actin Polymerization Promotes Breast Cancer Cells Migration and Invasion
}

\author{
Lei Lang ${ }^{\mathrm{a}}$ Yixuan Hou a,b Yanlin Chen ${ }^{\mathrm{a}}$ Gang Tuc Jing Tao ${ }^{\mathrm{d}}$ \\ Dan Yang ${ }^{a}$ Lei Xia Lixin Fu ${ }^{a}$ Kexin Sun ${ }^{a}$ Jiali Yin ${ }^{a} \quad$ Rui Chen ${ }^{c}$ \\ Meixi Penga Shuiqing Liu ${ }^{a} \quad$ Manran Liu $^{a}$
}

aKey Laboratory of Laboratory Medical Diagnostics, Chinese Ministry of Education, Chongqing Medical University, Chongqing, 'bxperimental Teaching Center of Basic Medicine Science, Chongqing Medical University, Chongqing, 'Department of Endocrine and Breast Surgery, the First Affiliated Hospital of Chongqing Medical University, Chongqing, ${ }^{\mathrm{d} D e p a r t m e n t}$ of Rheumatology and Immunology, Children's Hospital of Chongqing Medical University, Chongqing, China

\section{Key Words}

ATM • Cortactin $\cdot$ Hypoxia $\cdot$ Phosphorylation $\bullet$ Invasion

\begin{abstract}
Background/Aims: The ataxia-telangiectasia mutated (ATM) protein kinase is critical for the maintenance of genomic stability and acts as tumor suppressor. Although evidence shows that a DNA damage-independent ATM (oxidized ATM) may be involved in cancer progression, the underlying mechanism is still unclear. Methods: Immunohistochemistry, immunofluorescence and western blotting were applied to detect the levels of oxidized ATM. Transwell assay was used to detect the cell migration and invasion abilities in different treatments. Quantitative phosphoproteome analysis was performed using hypoxic BT549 cells, in the presence or absence of Ku60019, a specific inhibitor of ATM kinase. The phosphorylated cortactin, the target protein of oxidized ATM, was confirmed by immunoprecipitation-western blots and in vitro kinase assay. The functions of phosphorylated cortactin were studied by specific short hairpin RNA, site-directed mutation, transwell assay, and actin polymerization assay. Results: Enhanced oxidized ATM proteins were present not only in the advanced and invasive breast tumor tissues but also malignant hypoxic breast cancer cells, in the absence of DNA damage. Loss of ATM expression or inhibiting oxidized ATM kinase activity reduced breast cancer cell migration and invasion. Using quantitative phosphoproteomics approach, 333 oxidized ATM target proteins were identified, some of these proteins govern key signaling associated with gap junction, focal adhesion, actin cytoskeleton rearrangement. Cortactin, one of the

L. Lang and Y. Hou contributed equally to this work.


biggest changed phospho-protein, is a novel oxidized ATM-dependent target in response to hypoxia. Mechanically, we reveal that hypoxia-activated ATM can enhance the binding affinity of cortactin with Arp2/3 complex by phosphorylating cortactin at serine 113 , and as a result, in favor of breast cancer cell migration and invasion. Conclusion: Oxidized ATM can phosphorylate cortactin at serine 113, playing a critical role in promoting breast tumor cell mobility and invasion via actin polymerization.

\section{Introduction}

Breast cancer is the second leading cause of cancer death in women worldwide. In breast cancer patients diagnosed with distant metastasis, however, the 5-year survival rate is less than 33\% [1]. Like most solid tumors, tumor invasion and metastasis are the major causes of high mortality rate of breast cancer. Interestingly, a common feature of most solid tumors is low-oxygen tension or hypoxia [2]. Hypoxia is strongly associated with tumor cell proliferation, survival, metabolism, metastasis and angiogenesis [3-5]. It is also related to drug resistance and is considered as an independent poor prognostic indicator in various tumor types [6-8]. However, the molecular mechanisms of hypoxia accelerating breast cancer cell migration and invasion are still obscure.

Ataxia Telangiectasia (A-T) is a rare, human, recessive genetic disorder, involving complex multisystem defects characterized by cerebellar neurodegeneration, immunological abnormalities and an increased predisposition to cancer [9], caused by mutations in the ATM (Ataxia Telangiectasia mutated) gene. ATM kinase plays a critical role in DNA damage response (DDR) and the maintenance of genomic stability [10]. ATM is rapidly activated in response to DNA double-strand breaks (DSBs) by phosphorylating lots of key proteins, and is associated with cancer suppression [11]. However, this is just one aspect of ATM's function. Over the past few years, evidence shows that ATM is part of several signaling pathways, including cell growth [12] and metabolism [13], protein synthesis [14], autophagy [15], glucose uptake [16] and mitophagy [17], which are significantly activated in cancer cells. Stagni V et al. also demonstrated a tumorigenic role of ATM in breast cancer progression [18]. Furthermore, ATM kinase can also be activated by hypoxia in the absence of DNA damage, independent of Mre11-Rad50-NBS1 (MRN) complex from DSBs [19], which can thus be called DNA damageindependent ATM or oxidized ATM in these cases. However, the substrates of oxidized ATM following hypoxia-mediated activation and their associated signaling remain unknown.

The cortactin protein is encoded by CTTN gene, located at the 11q13 of chromosome 11. Cortactin is frequently overexpressed in advanced and invasive cancers [20]. Cortactin has many different functions including cytoskeleton rearrangement, cell motility, tumor cell invasion and metastasis [21]. Cortactin is also a phosphorylated protein that is originally identified as a substrate for Src tyrosine kinase [22]. In particular, the phosphorylation of Tyr residue in cortactin is required for cancer cell migration and metastasis [23, 24]. Recent studies showed that cortactin can also be phosphorylated by serine/threonine kinase extracellular regulated kinase 1/2 (ERK1/2), p21 activated kinase 1 (PAK1) and protein kinase D (PKD) [25-27]. In addition, only a few of the novel phosphorylation sites of cortactin protein have been identified [28], but the regulatory kinase and functions of those novel sites are still unclear.

In this research, we demonstrated a novel mechanism of breast cancer cell migration and invasion regulated by hypoxia-mediated ATM activation. Hypoxia can activate ATM independent of DNA DSBs (oxidized ATM) in pro-migration and invasion of breast cancer cells. Using quantitative phosphoproteome analysis, a vast number of oxidized ATM targets, including cortactin, were identified. These changed phosphorylated proteins may be involved in regulating actin cytoskeleton signaling. Furthermore, oxidized ATM phosphorylated cortactin on serine 113 is involved in Arp2/3 complex-mediated actin polymerization to promote breast cancer cells migration and invasion. 


\section{Cellular Physiology Cell Physiol Biochem 2018;51:2972-2988 \begin{tabular}{ll|l} 
and Biochemistry & $\begin{array}{l}\text { DOI: 10.1159/000496048 } \\
\text { Published onlIne: 14 December } 2018\end{array}$ & $\begin{array}{l}\text { C } 2018 \text { The Author(s). Published by S. Karger AG, Basel } \\
\text { www.karger.com/cpb }\end{array}$ \\
\hline
\end{tabular} \\ Invasion}

\section{Materials and Methods}

\section{Tissue samples}

Human breast tumor tissues and their corresponding normal breast tissues were collected from patients with breast tumor undergoing surgery at the first affiliated hospital of Chongqing Medical University. None of the patients had previously received radiotherapy or chemotherapy treatment. The investigation was approved by the ethics committee of Chongqing Medical University.

\section{Cell culture, treatments and transfections}

Human breast cancer cell lines BT549 and MDA-MB-231 were obtained from ATCC. BT549, MDAMB-231 and 293T cells were cultured in RPMI-1640 medium (Gibco) or Dulbecco's Modified Eagle's Medium (DMEM, Gibco) supplemented with $10 \%$ fetal bovine serum (FBS, Gibco), separately. All cells were incubated in a humidified atmosphere of $5 \% \mathrm{CO}_{2}$ at $37^{\circ} \mathrm{C}$. Hypoxia treatments of cells were performed in a tri-gas incubator (Thermo, USA) flushed with a gas mixture of $1 \% \mathrm{O}_{2}, 5 \% \mathrm{CO}_{2}$ and $94 \%$ nitrogen. For treatment of Ku60019 (S1570, Selleck Chemicals), an specific oxidized ATM inhibitor, cells were grown to approximately $60 \%$ confluence, the culture medium was replaced by fresh medium containing $1 \mu \mathrm{M}, 3 \mu \mathrm{M}$, $5 \mu \mathrm{M}$ Ku60019 or vehicle for 6 hours. The transfections of the indicated plasmids into cells were performed as described previously [29].

\section{shRNA and plasmids}

The lentivirus expression vector of ATM, cortactin shRNA and its infective lentivirus were obtained from GenePharma (Shanghai, China). The target sequences for ATM, cortactin, and negative control shRNA sequences are listed in Supplementary Table 1 (For all supplemental material see www.karger. com/10.1159/000496048/). pcDNA-Flag-CTTN, pCMV-CTTN (WT), pCMV-CTTN mutant (S113A and S113D) constructs were generated by GenePharma (Shanghai, China), and the sequences of resulting mutant cortactin were verified by direct sequencing. The pcDNA3-Flag-ATM construct was obtained from Addgene.

\section{RNA extraction and quantitative real-time PCR ( $q R T-P C R$ )}

Total RNA was isolated using Trizol (Invitrogen), following the manufacturer's instructions. The purified RNA was subjected to reverse transcription reactions using the PrimeScript RT Reagent Kit (TaKaRa, Dalian, China). qRT-PCR assay was performed using SYBR Premix Ex Taq II (TaKaRa, Dalian, China) according to the manufacturer's instructions. $\beta$-actin was used as internal reference gene. The used primers in qRT-PCR are listed in Supplementary Table 1 . Relative gene expression was measured as $2^{\mathrm{ct} \text { (internal control)-ct (gene). All }}$ experiments were performed at least 3 times.

\section{Western blot analysis and immunoprecipitation (IP)}

Western blotting assays and immunoprecipitation were performed as described previously [30]. The specific primary antibodies used are: ATM rabbit monoclonal antibody (2873, CST, 1:1000), p-ATM (s1981) rabbit monoclonal antibody (5883, CST, 1:1000), AKT rabbit monoclonal antibody (4685, CST, 1:1000), p-AKT (s473) mouse monoclonal antibody (12694, CST, 1:1000), $\gamma$-H2AX (s139) rabbit monoclonal antibody (9718, CST, 1:1000), $\beta$-Actin mouse monoclonal antibody (AA128, Beyotime, 1:1000), ARP3 rabbit monoclonal antibody (4738, CST, 1:1000), cortactin rabbit monoclonal antibody (3503, CST, WB:1:1000, IP:1:100), p-ATM/ATR Substrate Motif [(pS/pT) QG] MultiMab ${ }^{\text {TM }}$ rabbit monoclonal antibody mix (6966, CST, WB:1:1000, IP:1:100), p-cortactin (s113) rabbit polyclonal antibody (Zoonbio, 1:1000). The appropriate horseradish peroxidases (HRP)-conjugated secondary antibodies (Beyotime) were subsequently applied, and immunodetection was performed using the enhanced chemiluminescence system (Cool-Imager).

\section{IHC staining, immunofluorescence (IF) and F-actin staining}

Immunohistochemistry (IHC) staining was performed as described previously [31]. Briefly, the sections were incubated with a primary p-ATM (s1981) rabbit monoclonal antibody (ab81292, Abcam, 1:100), $\gamma$-H2AX (s139) rabbit monoclonal antibody (9718, CST, 1:250), overnight at $4^{\circ} \mathrm{C}$, and then 1 hour at room temperature, followed by the secondary antibody (PV-9000, ZSBIO, 1:100). The pathology scoring of the tissues was performed as described previously [31]. The expression of p-ATM and $\gamma$-H2AX were graded from 0 to $3+(0$, no staining; $1+, 1 \%-25 \% ; 2+, 26 \%-50 \% ; 3+,>50 \%$ staining). The staining intensities of 


\section{Cellular Physiology Cell Physiol Biochem 2018;51:2972-2988 and Biochemistry \begin{tabular}{l|l} 
DOI: 10.1159/000496048 & (c) 2018 The Author(s). Published by S. Karger AG, Basel \\
www.karger.com/cpb
\end{tabular} \\ Lang et al.: ATM Phosphorylating Cortactin Contributes to Breast Cancer Migration and Invasion}

p-ATM and $\gamma$-H2AX were assessed by examining $80 \%$ of the cell population. Images were captured using a Nikon Eclipse 80i microscope (Eclipse 80i, Tokyo, Japan).

Immunofluorescence (IF) was performed as described previously [32]. BT549 and MDA-MB-231 cells were cultured on prepared coverslips in hypoxia or normoxia to $70 \%$ confluence and then fixed within 4\% paraformaldehyde, and incubated overnight with primary p-ATM (s1981) mouse monoclonal antibody (ab19304, Abcam, 1:100) at $4^{\circ} \mathrm{C}$. Then the coverslips were incubated with a FITC-labeled goat anti-mouse secondary antibody (ab6785, Abcam, 1:1000) at room temperature (RT) for 1 hour. IF images were captured using a Nikon Eclipse 80i microscope (Eclipse 80i). Images were processed using NIS-Elements D software.

F-actin staining was performed using CytoPainter, Phalloidin-iFluor 594 Reagent (ab176757, Abcam), following the manufacturer's instructions. The cells were incubated with $4 \%$ paraformaldehyde at room temperature for 15 minutes, then stained with phalloidin-ifluor reagent (ab176757, Abcam, 1:1000) at RT for 1 hour. Images were captured using a Nikon Eclipse 80i microscope (Eclipse 80i). Quantitation of cortical polymerization of F-actin was performed with Imaris 7.4.0. (Bitplane) as described previously [33]. Intensity plots of signal intensity (y-axis) against distance in $10 \mu \mathrm{m}$ (x-axis), where each signal peak represents a F-actin.

\section{Cell migration and invasion assay}

Cell migration and invasion assays were performed using the $8 \mu \mathrm{m}$-pore size of Boyden chambers (Millipore, Darmstadt, Germany) as described previously [34]. Briefly, $2 \times 10^{4}$ of BT549 and MDA-MB-231 cells were seeded in a Boyden chamber coated with 1:7.5 diluted Matrigel (356231, Corning BioCoat, Bedford, USA) (for invasion assay) or without (for migration assay). Cells were incubated in hypoxic condition for 12 hours (for invasion assay) or 8 hours (for migration assay), then stained with crystal violet in methanol. The invaded and migrated cells were counted in five randomly selected visible views. Images were captured using a Nikon Eclipse 80i microscope (Eclipse 80i).

\section{In vitro kinase assay}

293T cells were transfected with pcDNA3-Flag-ATM or pcDNA3-Flag-Cortactin constructs. The 293T cells transfected with pcDNA3-Flag-Cortactin cultured in normoxia. The 293T cells transfected with pcDNA3Flag-ATM were treated with or without $5 \mu \mathrm{M}$ Ku60019 under normoxia or hypoxia condition, respectively. Then, Flag-ATM and Flag-Cortactin were immunopurified from the extracts with Anti-FLAG ${ }^{\circledR}$ M2 Magnetic Beads (M8823, Sigma) as described previously [35]. Kinase reactions were initiated by incubating purified ATM with purified Cortactin in kinase buffer provided by Amplite ${ }^{\mathrm{TM}}$ Universal Fluorimetric Kinase Assay Kit *Red Fluorescence* (AAT Bioquest). After reaction, the produced ADP was measured by Amplite ${ }^{\mathrm{TM}}$ Fluorimetric Kinase Assay Kit according to the manufacturer's manuals, using NOVOstar microplate reader (BMG LabTech) at Ex540/Em590 for $30 \mathrm{~min}$ and proteins were immunoblotted with the antibodies to cortactin and p-cortactin (s113).

\section{Actin polymerization assay}

Effects of cortactin phosphorylation at serine 113 on actin polymerization were assessed using Actin Polymerization Biochem Kits (BK003, Cytoskeleton, Denver, CO) following the manufacturer's instructions. Briefly, BT549 and MDA-MB-231 cells were transfected with Wild-type (WT), S113A and S113D mutant cortactin constructs and cultured in hypoxia for 2 days, cell lysate were collected and centrifuged at $150,000 \mathrm{~g}$ for 1 hour at $4^{\circ} \mathrm{C}$ to collect the clear supernatant, respectively. The cell lysates with equal amounts of proteins were immediately added to the final reaction mix containing ATP, pyrene actin (final conc. is $0.4 \mathrm{mg} / \mathrm{mL}$ ) in actin polymerization buffer. The kinetics of actin polymerization were monitored by fluorescence intensity until the fluorescent signal plateaus using a NOVOstar microplate reader with an excitation filter at $355 \mathrm{~nm}$ and an emission filter at $405 \mathrm{~nm}$, and the linear regression analysis was performed using Microsoft Excel.

\section{Quantitative phosphoproteomic analysis and bioinformatics analysis}

Quantitative phosphoproteomic analysis and bioinformatics analysis were performed via PTM-BIO Company, and the detailed protocols are available in the supplementary materials and methods. Briefly, the whole experiment consists of the following parts: sample preparation, protein extraction, trypsin digestion, TMT labeling (repeat once), HPLC fractionation, affinity enrichment, LC-MS/MS analysis, database search, bioinformatics analysis. 


\section{Cellular Physiology Cell Physiol Biochem 2018;51:2972-2988 \\ \begin{tabular}{ll|l} 
and Biochemistry & $\begin{array}{l}\text { DOI: 10.1159/000496048 } \\
\text { Published onlIne: 14 December } 2018\end{array}$ & $\begin{array}{l}\text { C) } 2018 \text { The Author(s). Published by S. Karger AG, Base } \\
\text { www.karger.com/cpb }\end{array}$ \\
\hline
\end{tabular} \\ Invasion}

\section{Statistical analysis}

Statistical analysis was performed using SPSS standard version 17.0. The data were presented as the mean \pm SD from at least three independent experiments. ANOVA followed by the student-Newman-Keuls multiple comparisons test was used to compare between multiple groups, and Student's $t$ test was used to compare between two groups. $P$-value $<0.05$ was considered statistically significant.

\section{Results}

Oxidized ATM promotes hypoxic breast cancer cell migration and invasion

Hypoxia is a common feature in all solid tumors and is associated with tumor development, malignant progression and metastatic outgrowth. Recently, it was proposed that ATM can be activated by hypoxia (called oxidized ATM), which is independent of DNA double-stranded breaks response manner [19]. To check whether oxidized ATM plays a key role in tumor progress, the immunohistochemistry was applied on normal breast tissue, invasive ductal carcinoma tissues without or with metastasis. We found that the levels of phospho-ATM (s1981) were negative or very low in normal breast tissues. Strong positive staining was gradually increased from invasive ductal carcinoma tissues without metastasis to those with metastasis. However, the levels of phospho-H2AX (s139), a DNA damage biomarker, were negative or very low in all examined tissues (Fig. 1A and Table 1). The levels of p-ATM (s1981) in BT549 and MDA-MB-231 cells were significantly increased following exposure of $1 \% \mathrm{O}_{2}$ (Fig. 1B-1C), no $\gamma$-H2AX was detected (Fig. 1C), suggesting an activated oxidized ATM in tumor cells. In addition, we observed enhanced migration (Fig. 1D) and invasion (Fig. 1E) of hypoxic BT549 and MDA-MB-231 cells in comparison with normoxic cells.

It was previously shown that altered ATM is related with cell migration and invasion [36]. In agreement with this observation, shRNA-mediated ATM knockdown (Fig. 2A-2B) suppressed cell migration and invasion in normoxic BT549 cells (Fig. 2C-2D, left upper panel). Similar effects (Fig. 2C-2D, right upper panel) were observed in these cells when inhibition of oxidized ATM using Ku60019 (Fig. 2E). Most importantly, hypoxia-induced migration and invasion were dramatically suppressed when ATM was knocked down or loss of oxidized ATM activity (Fig. 2C-2D, down panel). The same trend was also observed in other breast cancer cell MDA-MB-231 (data not show). Taken together, these data suggest that oxidized ATM plays a crucial role in promoting migration and invasion of breast cancer cells under hypoxia, which is independent of DNA DSBs.

Oxidized ATM-dependent phosphorylation contributes to the development and progression of breast cancer

Activated ATM can phosphorylate many targets in response to DNA damage or sustaining intracellular redox homeostasis [37]. To understand whether oxidized ATM is involved in activation and function of downstream targets and signaling, phosphoproteome analysis was performed. Under hypoxic condition, cell lysates from Ku60019-treated and non-treated BT549 cells were used in high-resolution LC-MS/MS analysis to identify the phosphorylation proteins of oxidized ATM (Fig. 3A). Altogether, 3697 phosphosites in 1596 proteins were identified, among which 2321 sites in 1145 proteins were quantified (Fig. 3B and Supplementary Table 2). Quantification ratio more than 1.5 was considered up-regulated and less than 0.67 was considered down-regulated in the analysis of phosphorylated proteins. There were 243 up-regulated phosphorylated sites distributing across 141 proteins, and 249 down-regulated phosphorylated sites distributing across 192 proteins in hypoxic BT549 cells treated with or without Ku60019 (Fig. 3B). Gene Ontology (GO) and KEGG Pathway analysis revealed that these changed phosphorylated proteins were most likely involved in biological processes associated with cell-cell adhesion, cell proliferation, protein modification and 
Fig. 1. Hypoxia activates oxidized ATM kinase to promote breast cancer cells migration and invasion. (A) Immunohistochemistry determination of p-ATM (s1981) and $\gamma$-H2AX (s139) expressions in normal mammary epithelium and breast carcinomas. The representative photographs of IHC staining are shown. Scale bars, $100 \mu \mathrm{m}$. (B, C) The p-ATM and total ATM expressions were tested in nomoxic $\left(\begin{array}{ll}21 \% & \mathrm{O}_{2}\end{array}\right)$ or hypoxic $\left(\begin{array}{ll}1 \% & \mathrm{O}_{2}\end{array}\right)$ breast cancer cells BT549 and MDA-MB-231 using immunofluorescence staining (scale bars, $10 \mu \mathrm{m})(\mathrm{B})$ and western blotting (C). (D, E) Cell migration and invasion potentials of nomoxic and hypoxic BT549 and MDA-MB-231 cells were determined by transwell analysis. Scale bars, $50 \mu \mathrm{m}$.

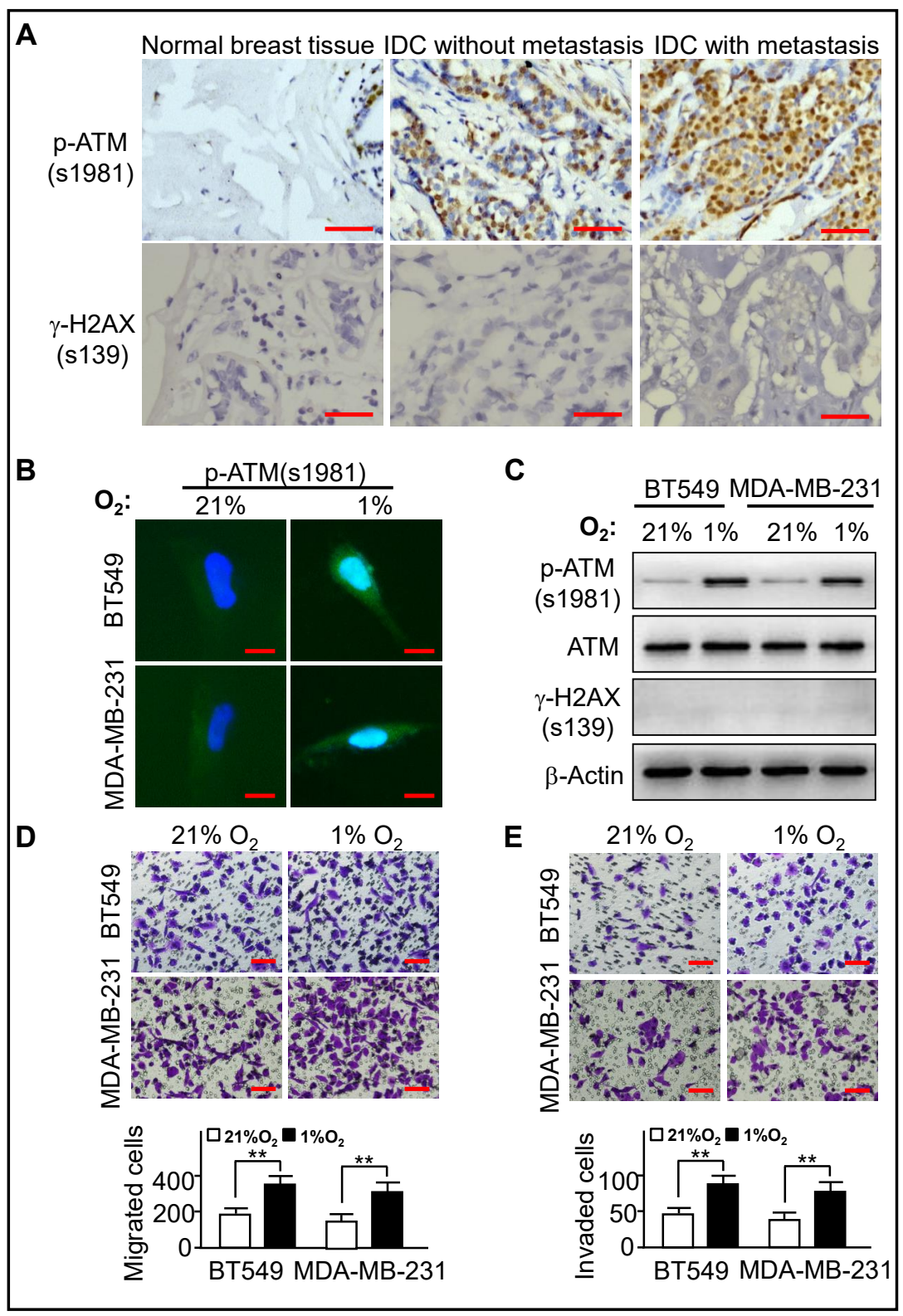

Table 1. Summary of IHC staining of p-ATM and $\gamma$-H2AX in breast tissues

\begin{tabular}{lcccc}
\hline \multicolumn{1}{c}{ Type } & \multicolumn{2}{c}{ p-ATM (s1981) } & \multicolumn{2}{c}{$\gamma$-H2AX (s139) } \\
& Negative N (\%) & Positive N (\%) & Negative N (\%) & Positive N (\%) \\
\hline Normal breast tissue (n=15) & $14(93.33)$ & $1(6.67)$ & $14(93.33)$ & $1(6.67)$ \\
IDC without metastasis (n=23) & $10(43.47)$ & $23(56.53)$ & $20(86.96)$ & $3(13.04)$ \\
IDC with metastasis (n=26) & $7(26.92)$ & $19(73.07)$ & $22(84.32)$ & $4(15.38)$ \\
\hline
\end{tabular}




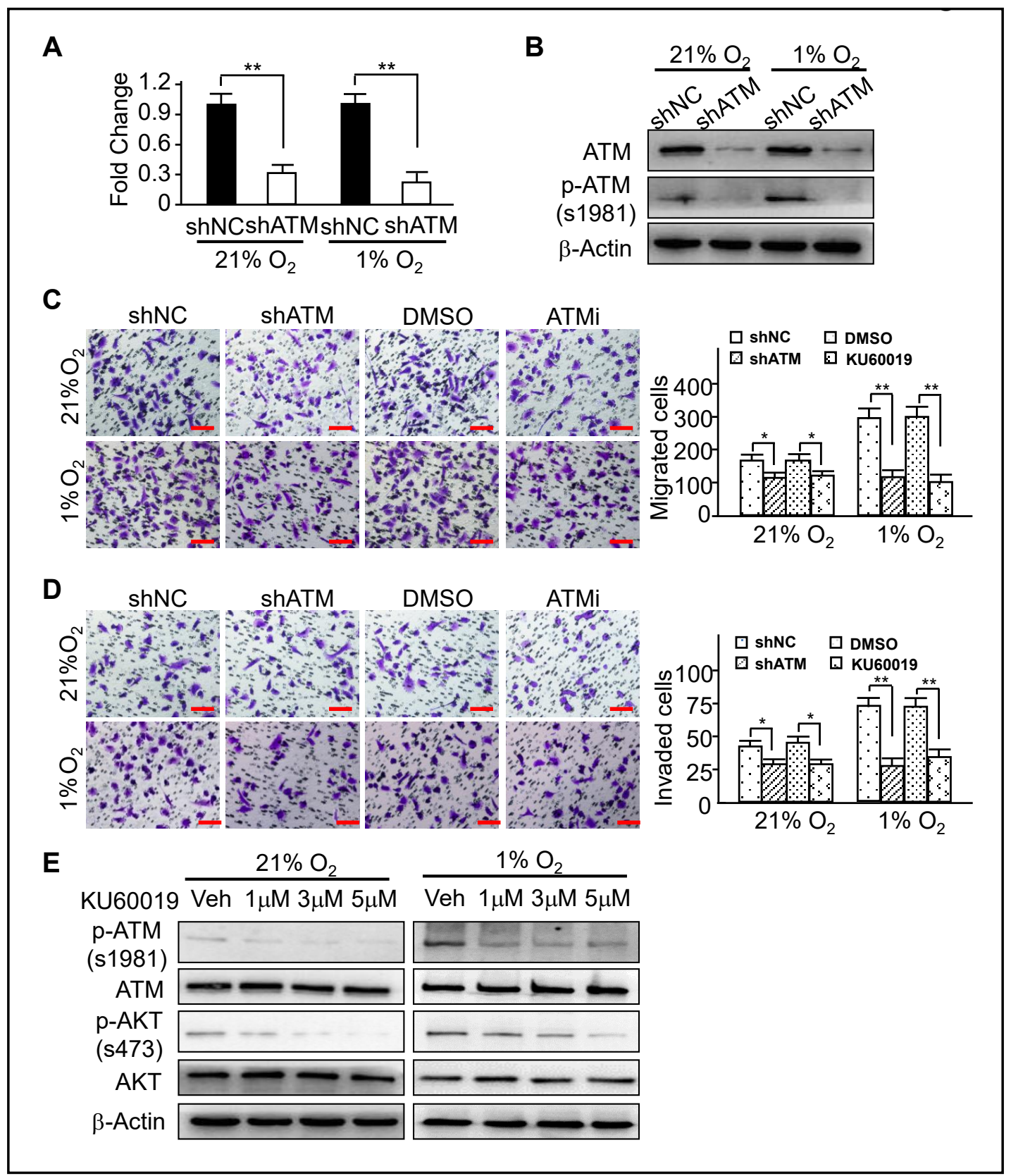

Fig. 2. Oxidized ATM is required for cell migration and invasion in breast cancer cells. (A, B) The knocked down efficiency of ATM by specific shRNA was measured in normoxic and hypoxic breast cancer cells BT549 using qRT-PCR (A) and western blot (B) (**, p<0.01). (C, D) BT549 cells were stably knocked down ATM or treated with Ku60019 (labeled as ATMi), the cell migration (C) and invasion (D) potentials were tested by transwell assay in normoxia and hypoxia, scale bars, $50 \mu \mathrm{m}(* *, p<0.01)$. (E) BT549 cells were treated with or without an oxidized ATM inhibitor Ku60019 (1 $\mu \mathrm{M}$ to $5 \mu \mathrm{M})$ for 6 hours in normoxia and hypoxia. The activation of oxidized ATM and AKT was measured by western blotting. 


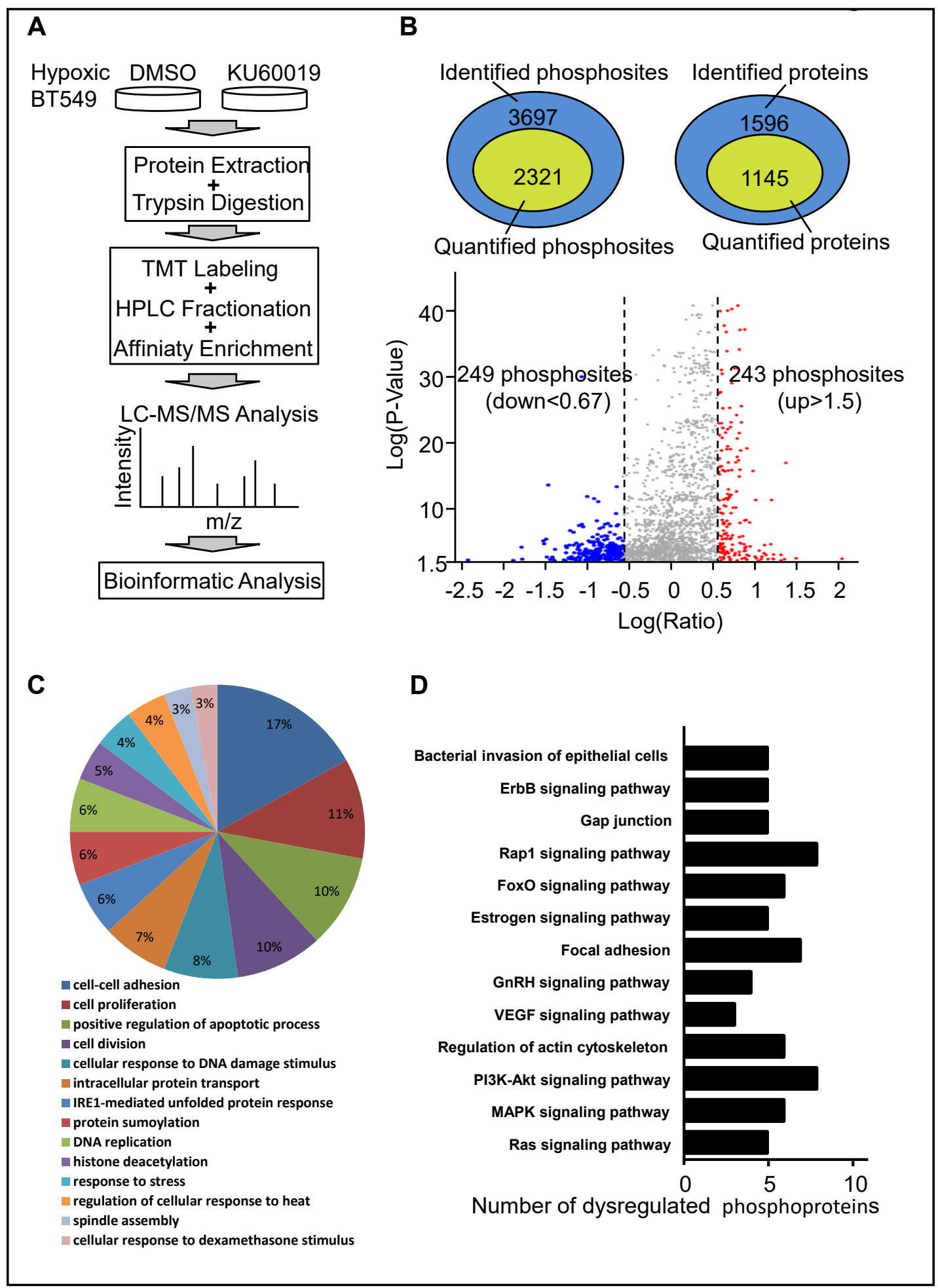

Fig. 3. Phosphoproteomic analysis to identify the downstream phosphorylated proteins of oxidized ATM in breast tumor cell treated with or without Ku60019. (A) Cartoon depicting the phosphoproteomic analysis workflow. (B) Numbers of the identified total phosphosites of proteins and phosphoproteins, quantified phosphosites of proteins and phosphoproteins. (C) GO classification of phosphoproteins based on biological process. (D) The significant pathways based on the changed phosphoproteins in hypoxic BT549-DMSO vs BT549-ATMi cells were enriched by DAVID v6.7 using KEGG database.

\section{KARGER}




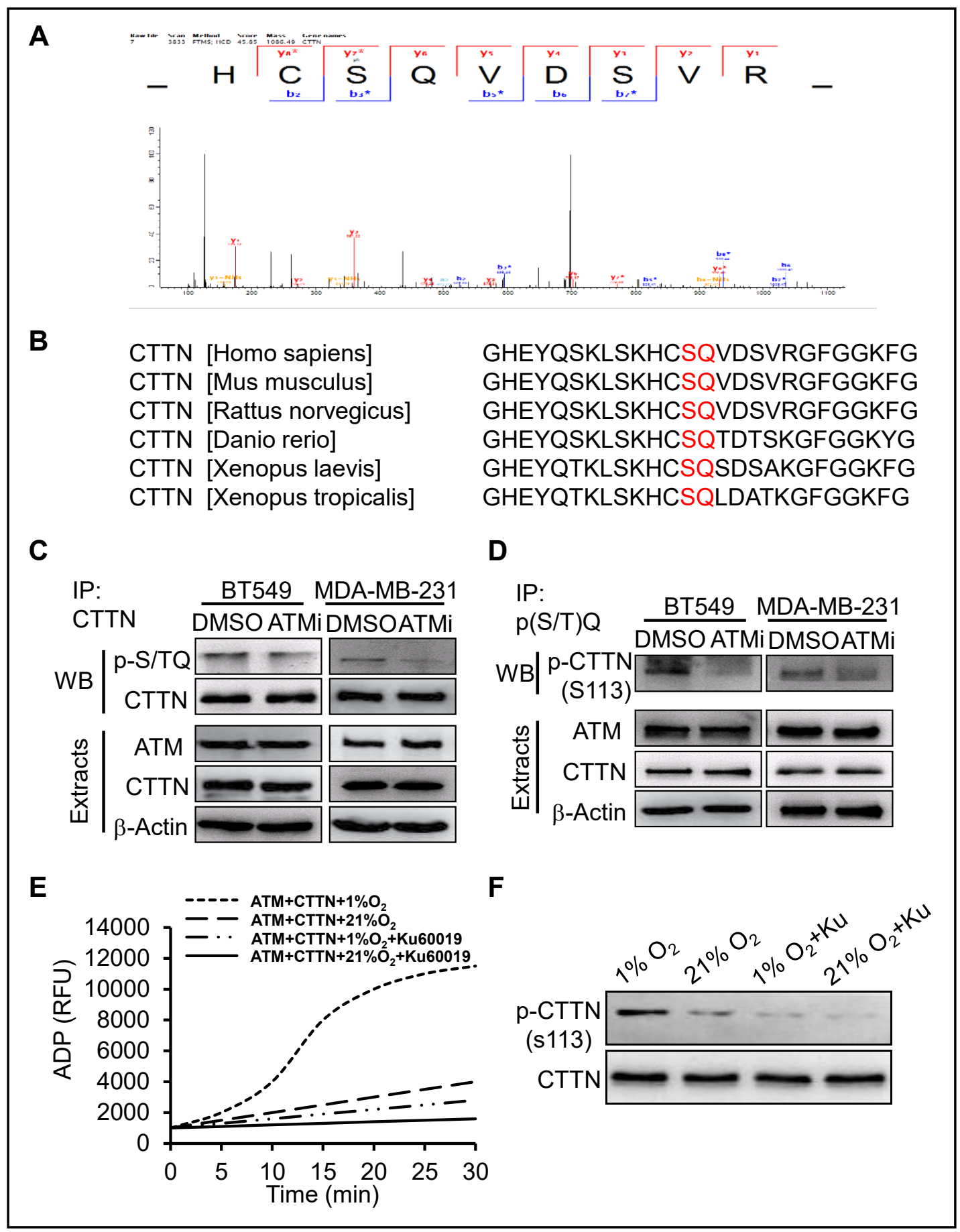

Fig. 4. Oxidized ATM phosphorylates cortactin on Serine 113. (A) Phosphorylated serine 113 residues were identified using LC-MS/MS. (B) The ATM phosphorylation consensus sequences of cortactin in human, mouse, rat, zebrafish, and xenopus. (C-D) Breast cancer cells BT549 and MDA-MB-231 were cultured in hypoxia condition with or without Ku60019 (labeled as ATMi), cell extracts were immunoprecipitated with cortactin antibody (C) or the specific ATM/ATR substrate p-(S/T)Q antibody (D), immunoblotting with the indicated antibodies to identify the cortactin serine 113 as a phosphorylated site of oxidized ATM. (E-F) Cell culture and treatment of 293T cells as described in Material part 2.8 in detail. In vitro kinase assay was performed using purified ATM kinase mixed with substrates of purified cortactin. The ADP products (E) and the phosphorylated cortactin (F) are shown. 


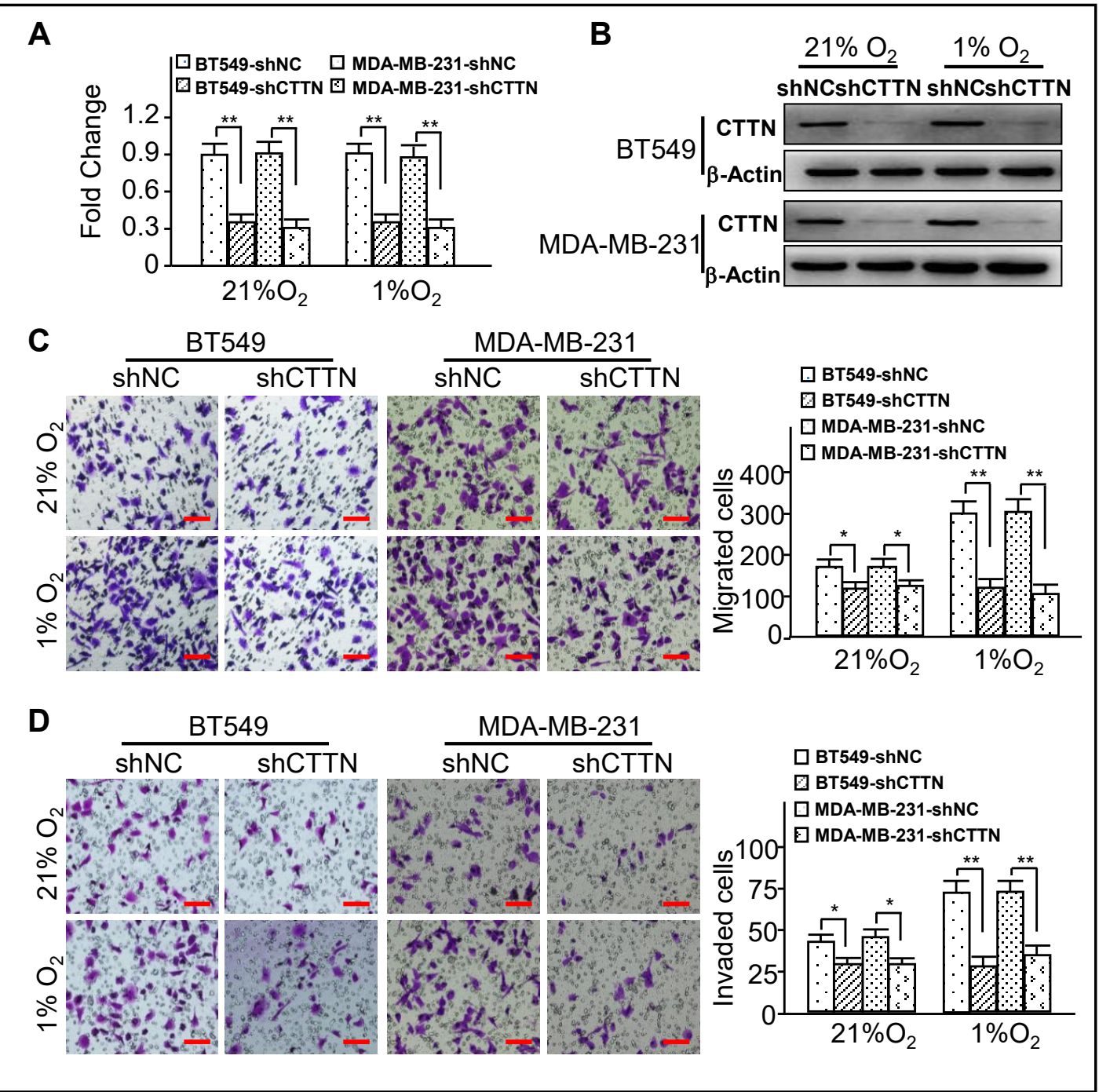

Fig. 5. Lentivirus-mediated knockdown of cortactin suppresses breast cancer cell migration and invasion. (A, B) The knocked down efficiencies of CTTN by the specific shRNA in normoxic and hypoxic BT549 and MDA-MB-231 cells were evaluated by qRT-PCR (A) and western blotting (B) (**, p<0.01). (C, D) Transwell assay was used to test cell migration (C) and invasion (D) abilities of BT549 and MDA-MB-231 cells with wild type or silenced CTTN in normoxia and hypoxia condition, scale bars, $50 \mu \mathrm{m}(* *, \mathrm{p}<0.01)$.

response to cellular stress other than DNA damage stimulus (Fig. 3C), which may be governed by gap junction, focal adhesion, actin cytoskeleton rearrangement signaling pathways (Fig. 3D). These data suggest that oxidized ATM-dependent phosphorylation contributes to the development and progression of breast cancer.

\section{ATM phosphorylates cortactin on serine 113}

Coractin is one of the most significantly changed phosphorylated protein in our phosphoproteome. Cortactin is a key member of protein involved in cytoskeleton organization and cell mobility $[38,39]$, suggesting that cortactin may play a role in oxidized ATM-induced breast cancer cell invasion. LC-MS/MS analysis unraveled that oxidized ATM phosphorylated cortactin on serine 113 (Fig. 4A), and inhibition of oxidized ATM led to a $60 \%$ reduction of cotactin phosphorylation in BT549 (data not shown). Interestingly, serine 113 , a typical (S/T)Q site on the cortactin protein, is the classic ATM specific substrate sequence which is highly conserved across human, mouse, rat, zebrafish, and xenopus (Fig.

\section{KARGER}


4B). To validate whether oxidized ATM could phosphorylate cortactin at serine 113 , we carried out the protein immunoprecipitation with an antibody recognizing cortactin or the ATM/ATR substrate motif $\mathrm{p}(\mathrm{S} / \mathrm{T}) \mathrm{Q}$ followed by immunoblotting with antibody against ATM/ ATR substrate motif $\mathrm{p}(\mathrm{S} / \mathrm{T}) \mathrm{Q}$ or the phosphorylated cortactin. The phosphorylation levels of cortactin were increased in response to hypoxia and decreased in Ku60019 treatment in both BT549 and MDA-MB-231 cells (Fig. 4C-4D). To further confirm that oxidized ATM has the ability to phosphorylate cortactin on serine 113, in vitro ATM kinase assay was performed. Oxidized ATM phosphorylated cortactin at serine 113 under hypoxia, and this phosphorylation could be attenuated by Ku60019 (Fig. 4E-4F), supporting a critical role of oxidized ATM in regulating cortactin phosphorylation in hypoxic breast cancer cells.

\section{ATM-mediated cortactin phosphorylation enhances breast cancer cell migration and} invasion

To evaluate the role of cortactin in breast cancer cell migration and invasion, a lentiviral vector encoding a shRNA specifically against cortactin was infected into BT549 and MDAMB-231 cells. Efficient knockdown of cortactin (Fig. 5A-5B) resulted in decreased cell migration and invasion in BT549 and MDA-MB-231 cells (Fig. 5C-5D). To further understand whether oxidized ATM-mediated cortactin phosphorylation at serine 113 plays a critical role in cancer cell migration and invasion, we used the specifically developed anti-phosphocortactin S113 antibody (p-CTTN S113) to determine the levels of phosphorylated cortactin. As expected, the phosphorylation levels of cortactin at serine 113 were dramatically decreased when knockdown of ATM or inhibition of oxidized ATM kinase activity (Fig. 6A), suggesting that oxidized ATM is required for cortactin phosphorylation at serine 113. In line with these findings, cortactin, S113A (a hypo-phosphorylated cortactin mutant), S113D (a hyperphosphorylated cortactin mutant) as well as WT cortactin were transfected into hypoxic BT549 and MDA-MB-231 cells (Fig. 6B). The obvious changes of migration and invasion potentials of BT549 and MDA-MB-231 cells (Fig. 6C-6D) were detected. In comparison with the parental cells, overexpression of WT cortactin endowed the BT549 and MDA-MB-231 cells with enhanced migration and invasion abilities in the presence of hypoxia. However, the S113A mutant cortactin led to a decreased potential of these breast cancer cells in migration and invasion compared with WT cortactin, and the S113D mutant cortactin counteracted these effects. Moreover, inhibiting oxidized ATM by Ku60019 resulted in reduced cancer cell migration and invasion, which can be rescued by ectopic expression of cortactin S113D mutant (Fig. 6C-6D, right panel). These data demonstrate that oxidized ATM-mediated phosphorylation of cortactin is essential for gaining the migration and invasion abilities of hypoxic breast cancer cells.

Oxidized ATM-mediated cortactin phosphorylation modulates actin polymerization

We then sought to elucidate molecular mechanisms of oxidized ATM-mediated cortactin phosphorylation in promoting hypoxic breast cancer cell migration and invasion. Previous studies have reported that cortactin can co-localize with Arp2/3 complex to promote nucleation of actin filaments $[40,41]$. To test whether oxidized ATM-mediated phosphorylated cortactin modulates the interaction of cortactin-Arp2/3 complex, co-immunoprecipitation assay was employed, which showed cortactin in the Arp2/3 immunocomplex under hypoxia (Fig 7A-7B). Importantly, cortactin-Arp2/3 interaction was significantly decreased under inhibition of oxidized ATM kinase activity by Ku60019 in either BT549 (Fig. 7A) or MDAMB-231 cells (Fig. 7B). To further assess whether phosphorylated cortactin at serine 113 mediates actin polymerization, we detected polymerization of actin in BT549 and MDAMB-231 cells with ectopic wild type cortactin (WT) or mutant cortactin (S113A, S113D), and the cells with shATM or treated with or without Ku60019, respectively. Inhibition of oxidized ATM kinase activity or knockdown of ATM expression, as well as overexpression of cortactin S113A mutant in BT549 and MDA-MB-231 cells attenuated F-actin formation (Fig. 7C-7D). However, ectopic S113D mutant cortactin enhanced F-actin formation compared with WT control (Fig. 7C-7D). We then performed in vitro actin polymerization assay, which 


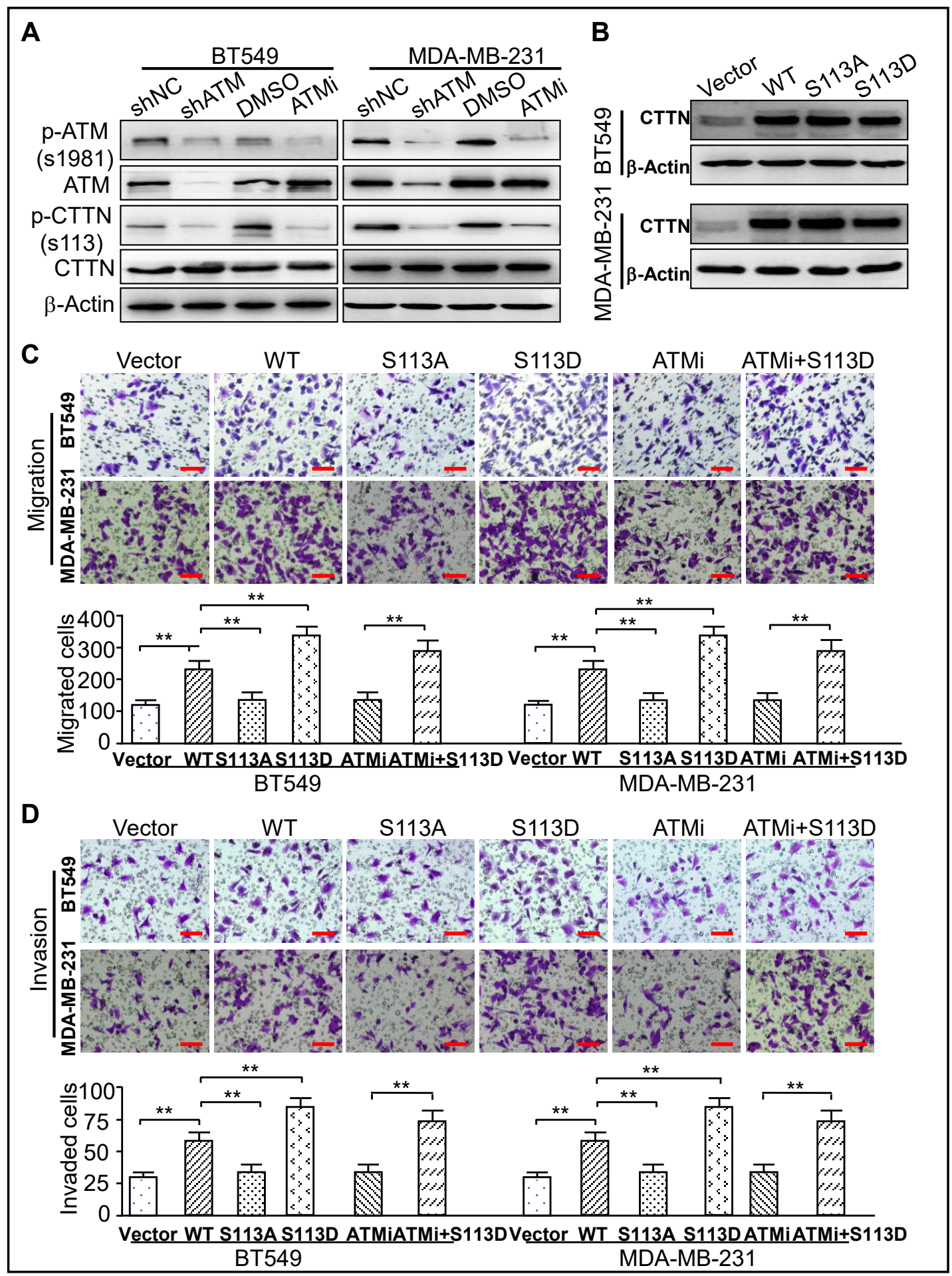

Fig. 6. Oxidized ATM-mediated phosphorylation of cortactin promotes breast cancer cells migration and invasion. (A) Extracts from hypoxic BT549 and MDA-MB-231 (shNC, shATM, DMSO and ATMi) cells were used to measure the protein levels of p-ATM (s1981), p-CTTN (s113), total ATM and CTTN by the indicated antibodies. (B) Western blotting to determine cortactin protein levels in hypoxic BT549 and MDA-MB-231 cells stably transfected with WT, mutant (S113A or S113D) cortactin and the control vector. $\beta$-Actin is the loading control. (C-D) Transwell assay to test cell migration (C) and invasion (D) of hypoxic BT549 and MDAMB-231 cells stably expressing WT, mutant cortactin (S113A or S113D) and control plasmid treated with or without Ku60019 (labeled as ATMi). Scale bars, $50 \mu \mathrm{m}(* *, \mathrm{p}<0.01)$. 
Lang et al.: ATM Phosphorylating Cortactin Contributes to Breast Cancer Migration and Invasion

Fig.7.Thephosphorylated cortactin binding with Arp2/3 promotes actin polymerization. (A, B) Cortactin was immunoprecipitated from extracts of BT549 (A) and MDA-MB-231 cells (B) treated with or without Ku60019 under hypoxia, followed by immunoblotting with indicated antibodies. The protein binding between cortactin and Arp2/3 is shown. (C, D) The polymerized actins (F-actin) were displayed by immunofluorescence staining using rhodamineconjugated phalloidin in hypoxic BT549 and MDA-MB-231 cells stably expressing WT, mutant (S113A or S113D) cortactin, shATM, or control cells treated with Ku60019 (labeled as ATMi). Scale bars, $10 \mu \mathrm{m}$. (E, F) Equal amounts of protein extracts from BT549 cells (E) and MDA-MB-231 cells (F) transfected with cortactin WT, S113A or S113D construct were incubated with pyrene actin monomers, and the kinetics of actin polymerization was measured as described in Materials and Methods. (G) A proposed model to show oxidized ATM in promoting breast cancer cells migration and invasion via phosphorylated-cortactin/ Arp2/3-mediated actin polymerization.

showed that S113D mutant and WT cortactin notably increased G-actin polymerization, whereas S113A mutant attenuated G-actin polymerization (Fig. 7E-7F). Collectively, our data demonstrate that Arp2/3 complex, in association with phosphorylated cortactin, can modulate actin polymerization, which consequently promotes breast cancer cell migration and invasion.

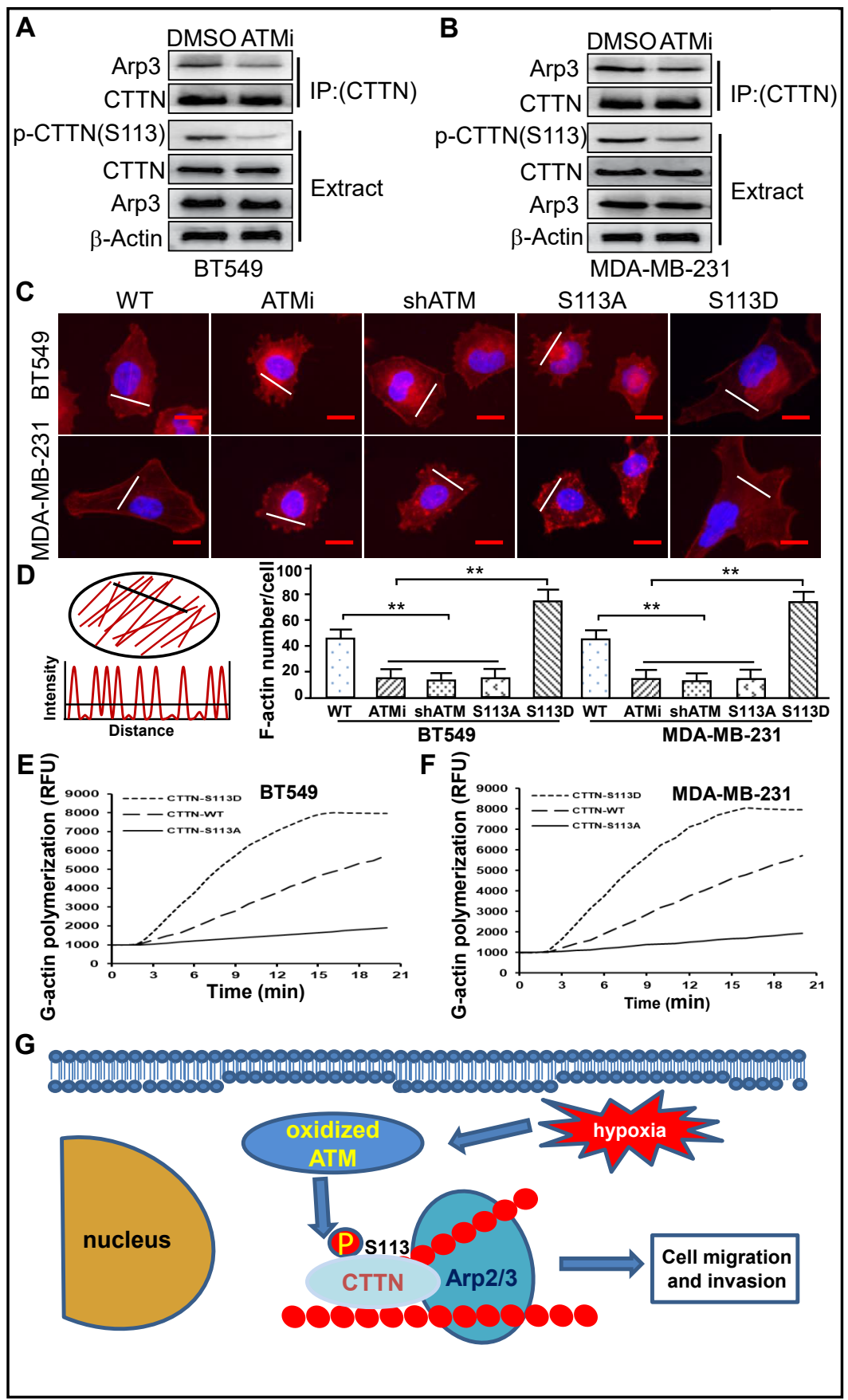




\section{Cellular Physiology Cell Physiol Biochem 2018;51:2972-2988

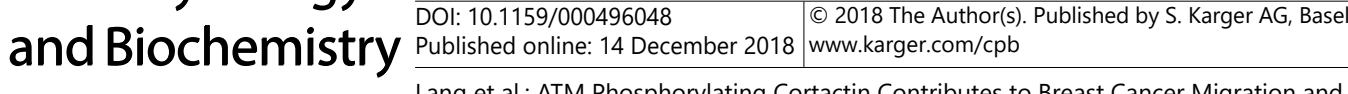 \\ Invasion}

\section{Discussion}

ATM, the protein kinase mutated in the rare human disease ataxia telangiectasia (A$\mathrm{T}$ ), has been classically considered to be a tumor suppressor gene. In this study, we have characterized a tumor promoting activity for DNA damage-independent ATM coxidized ATM). We showed that oxidized ATM is hyperactive in the invasive breast cancer tissues and under hypoxic conditions. We further demonstrated that the knockdown of ATM or inhibition of oxidized ATM activation correlates very well with attenuated breast cancer cells migration and invasion abilities. At the molecular level, we are the first to identify that the activation of oxidized ATM by hypoxia can phosphorylate cortactin at Serine 113 site, and the phosphorylated cortactin binding with Arp2/3 complex mediates actin polymerization to promote breast cancer cells migration and invasion (Fig. 7G).

ATM kinase is a major protector of genomic stability, which is attributable to its key role in DNA damage response (DDR) caused by double-stranded breaks [11]. However, other recent research suggested that ATM is also involved in many other cancer signaling pathways other than DNA damage response, which relates to chemoresistance, radioresistance, metastasis, cell growth and proliferation. Chemoresistance may be caused by DNA damage-independent ATM signaling via overexpression of HMGA proteins [42]. Oxidized ATM signaling, supports breast tumor metastasis through the regulation of IL-8 [36]. Oxidized ATM can also activate AKT to promote cell growth and proliferation in breast cancer associated fibroblasts [12]. In the study, we showed that oxidized ATM is aberrantly high in expression in breast cancer tissues with lymph-node metastasis (Fig. 1). And we also found that the activity and expression of oxidized ATM are essential for breast cancer cell migration and invasion (Fig. 2). Thus, we guessed that oxidized ATM may play a dual function in cancer, depending on the specific context.

The canonical mode demonstrated that the activated ATM, which is generally activated by the Mre11-Rad50-NBS1 (MRN) complex from DSBs, can phosphorylate more than 700 substrates that are involved in many of the signaling pathways to govern gene expression, cell cycle and DNA repairs [11]. Here, we found that oxidized ATM can be activated by hypoxia in the absence of DNA damage, which is consistent with the findings by Possik PA et al. [43]. In this study, we identified a set of oxidized ATM-associated phosphorylated proteins including cortactin, by employing a specific inhibitor of oxidized ATM kinase activity, Ku60019, to administer breast cancer cell BT549 under hypoxia. Bioinformatic analysis of the quantitative phosphoproteome data revealed that actin cytoskeleton organization-associated signaling is a major pathway regulated by hypoxia-activated oxidized ATM.

The functional cortactin in cell migration and invasion is regulated by phosphorylation. In some cases, the phosphorylation of cortactin by serine/threonine kinase, extracellular regulated kinase 1/2 (ERK1/2) and p21 activated kinase 1 (PAK1), has been shown to increase $\mathrm{N}$-WASP binding to cortactin $[25,26]$. In other cases, AMP-activated protein kinase (AMPK) phosphorylating cortactin modulates the interaction of cortactin and cortical-actin [44]. In this study, we demonstrated that hypoxia-activated oxidized ATM phosphorylates cortactin at serine 113 site increasing its binding affinity with Arp2/3 complex, thus enhancing actin polymerization and promoting breast cancer cell migration and invasion.

\section{Conclusion}

In conclusion, ATM can be activated under hypoxia independent of DNA DSBs. Hypoxiaactivated oxidized ATM, phosphorylates cortactin, which is, therefore, involved in actin cytoskeleton organization and actin polymerization to play an essential role in breast cancer cell migration and invasion. Our study provides a novel insight into ATM functions in tumor development. And these findings suggest that oxidized ATM and its target proteins may be a potential therapeutic target for tumor metastasis. 


\section{Cellular Physiology Cell Physiol Biochem 2018;51:2972-2988 \begin{tabular}{ll|l} 
and Biochemistry & $\begin{array}{l}\text { DOI: 10.1159/000496048 } \\
\text { Published onlIne: 14 December } 2018\end{array}$ & $\begin{array}{l}\text { C } 2018 \text { The Author(s). Published by S. Karger AG, Basel } \\
\text { www.karger.com/cpb }\end{array}$ \\
\hline
\end{tabular} \\ Invasion}

\section{Acknowledgements}

This work was supported in part by National Natural Science Foundation of China (NSFC 81472476, NSFC 31671481, and NSFC 31171336) to Manran Liu; and supported by National Natural Science Foundation of China (NSFC 81402180) to Yixuan Hou. We also appreciated Dr. Yong Teng, working at Georgia Cancer Center, Augusta University of USA, to give us suggestion and help for this study.

\section{Disclosure Statement}

None of the authors declare conflicts of interest.

\section{References}

-1 Jemal A, Ward EM, Johnson CJ, Cronin KA, Ma J, Ryerson B, Mariotto A, Lake AJ, Wilson R, Sherman RL, Anderson RN, Henley SJ, Kohler BA, Penberthy L, Feuer EJ, Weir HK: Annual report to the nation on the status of cancer, 1975-2014, featuring survival. J Natl Cancer Inst 2017;109

2 Endrich B, Reinhold HS, Gross JF, Intaglietta M: Tissue perfusion inhomogeneity during early tumor growth in rats. J Natl Cancer Inst 1979;62:387-395.

-3 Schmaltz C, Hardenbergh PH, Wells A, Fisher DE: Regulation of proliferation-survival decisions during tumor cell hypoxia. Mol Cell Biol 1998;18:2845-2854.

4 Liao J, Qian F, Tchabo N, Mhawech-Fauceglia P, Beck A, Qian Z, Wang X, Huss WJ, Lele SB, Morrison CD, Odunsi K: Ovarian cancer spheroid cells with stem cell-like properties contribute to tumor generation, metastasis and chemotherapy resistance through hypoxia-resistant metabolism. PLoS One 2014;9:e84941.

-5 Ko YS, Cho SJ, Park J, Choi Y, Lee JS, Youn HD, Kim WH, Kim MA, Park JW, Lee BL: Hypoxic inactivation of glycogen synthase kinase-3beta promotes gastric tumor growth and angiogenesis by facilitating hypoxiainducible factor-1 signaling. APMIS 2016;124:748-756.

-6 Zhao M, Zhang Y, Zhang H, Wang S, Zhang M, Chen X, Wang H, Zeng G, Chen X, Liu G, Zhou C: Hypoxiainduced cell stemness leads to drug resistance and poor prognosis in lung adenocarcinoma. Lung Cancer 2015;87:98-106.

7 Lin SC, Liao WL, Lee JC, Tsai SJ: Hypoxia-regulated gene network in drug resistance and cancer progression. Exp Biol Med (Maywood) 2014;239:779-792.

-8 Zhang ZG, Zhang QN, Wang XH, Tian JH: Hypoxia-inducible factor 1 alpha (hif-1alpha) as a prognostic indicator in patients with gastric tumors: A meta-analysis. Asian Pac J Cancer Prev 2013;14:4195-4198.

-9 Lavin MF, Gueven N, Bottle S, Gatti RA: Current and potential therapeutic strategies for the treatment of ataxia-telangiectasia. Br Med Bull 2007;81-82:129-147.

10 Lavin MF, Birrell G, Chen P, Kozlov S, Scott S, Gueven N: Atm signaling and genomic stability in response to DNA damage. Mutat Res 2005;569:123-132.

11 Shiloh Y, Ziv Y: The atm protein kinase: Regulating the cellular response to genotoxic stress, and more. Nat Rev Mol Cell Biol 2013;14:197-210.

12 Tang S, Hou Y, Zhang H, Tu G, Yang L, Sun Y, Lang L, Tang X, Du YE, Zhou M, Yu T, Xu L, Wen S, Liu C, Liu M: Oxidized atm promotes abnormal proliferation of breast cafs through maintaining intracellular redox homeostasis and activating the pi3k-akt, mek-erk, and wnt-beta-catenin signaling pathways. Cell Cycle 2015;14:1908-1924.

13 Tang S, Yang L, Tang X, Liu M: The role of oxidized atm in the regulation of oxidative stress-induced energy metabolism reprogramming of cafs. Cancer Lett 2014;353:133-144.

$\checkmark 14$ Yang DQ Kastan MB: Participation of atm in insulin signalling through phosphorylation of eif-4e-binding protein 1. Nat Cell Biol 2000;2:893-898.

15 Alexander A, Kim J, Walker CL: Atm engages the tsc2/mtorc1 signaling node to regulate autophagy. Autophagy 2010;6:672-673.

16 Halaby MJ, Hibma JC, He J, Yang DQ: Atm protein kinase mediates full activation of akt and regulates glucose transporter 4 translocation by insulin in muscle cells. Cell Signal 2008;20:1555-1563. 


\section{Cellular Physiology and Biochemistry}

Cell Physiol Biochem 2018;51:2972-2988

\begin{tabular}{l|c|c|}
\hline DOI: 10.1159/000496048 & C 2018 The Author(s). Published by S. Karger AG, Basel
\end{tabular}

Published online: 14 December 2018 www.karger.com/cpb

Lang et al.: ATM Phosphorylating Cortactin Contributes to Breast Cancer Migration and Invasion

17 Valentin-Vega YA, Kastan MB: A new role for atm: Regulating mitochondrial function and mitophagy. Autophagy 2012;8:840-841.

-18 Stagni V, Manni I, Oropallo V, Mottolese M, Di Benedetto A, Piaggio G, Falcioni R, Giaccari D, Di Carlo S, Sperati F, Cencioni MT, Barila D: Atm kinase sustains her2 tumorigenicity in breast cancer. Nat Commun 2015;6:6886.

19 Bencokova Z, Kaufmann MR, Pires IM, Lecane PS, Giaccia AJ, Hammond EM: Atm activation and signaling under hypoxic conditions. Mol Cell Biol 2009;29:526-537.

20 Schuuring E, Verhoeven E, Litvinov S, Michalides RJ: The product of the ems1 gene, amplified and overexpressed in human carcinomas, is homologous to a v-src substrate and is located in cell-substratum contact sites. Mol Cell Biol 1993;13:2891-2898.

-21 Kirkbride KC, Sung BH, Sinha S, Weaver AM: Cortactin: A multifunctional regulator of cellular invasiveness. Cell Adh Migr 2011;5:187-198.

22 Wu H, Reynolds AB, Kanner SB, Vines RR, Parsons JT: Identification and characterization of a novel cytoskeleton-associated pp60src substrate. Mol Cell Biol 1991;11:5113-5124.

23 Huang C, Liu J, Haudenschild CC, Zhan X: The role of tyrosine phosphorylation of cortactin in the locomotion of endothelial cells. J Biol Chem 1998;273:25770-25776.

24 Li Y, Tondravi M, Liu J, Smith E, Haudenschild CC, Kaczmarek M, Zhan X: Cortactin potentiates bone metastasis of breast cancer cells. Cancer Res 2001;61:6906-6911.

25 Martinez-Quiles N, Ho HY, Kirschner MW, Ramesh N, Geha RS: Erk/src phosphorylation of cortactin acts as a switch on-switch off mechanism that controls its ability to activate n-wasp. Mol Cell Biol 2004;24:52695280 .

-26 Grassart A, Meas-Yedid V, Dufour A, Olivo-Marin JC, Dautry-Varsat A, Sauvonnet N: Pak1 phosphorylation enhances cortactin-n-wasp interaction in clathrin-caveolin-independent endocytosis. Traffic 2010;11:1079-1091.

-27 Eiseler T, Hausser A, De Kimpe L, Van Lint J, Pfizenmaier K: Protein kinase d controls actin polymerization and cell motility through phosphorylation of cortactin. J Biol Chem 2010;285:18672-18683.

-28 Martin KH, Jeffery ED, Grigera PR, Shabanowitz J, Hunt DF, Parsons JT: Cortactin phosphorylation sites mapped by mass spectrometry. J Cell Sci 2006;119:2851-2853.

29 Yang L, Hou Y, Yuan J, Tang S, Zhang H, Zhu Q, Du YE, Zhou M, Wen S, Xu L, Tang X, Cui X, Liu M: Twist promotes reprogramming of glucose metabolism in breast cancer cells through pi3k/akt and p53 signaling pathways. Oncotarget 2015;6:25755-25769.

30 Wu K, Liu M, Li A, Donninger H, Rao M, Jiao X, Lisanti MP, Cvekl A, Birrer M, Pestell RG: Cell fate determination factor dach1 inhibits c-jun-induced contact-independent growth. Mol Biol Cell 2007;18:755767.

-31 Zhang H, Hou Y, Xu L, Zeng Z, Wen S, Du YE, Sun K, Yin J, Lang L, Tang X, Liu M: Cytoplasmic drosha is aberrant in precancerous lesions of gastric carcinoma and its loss predicts worse outcome for gastric cancer patients. Dig Dis Sci 2016;61:1080-1090.

-32 Wang L, Hou Y, Sun Y, Zhao L, Tang X, Hu P, Yang J, Zeng Z, Yang G, Cui X, Liu M: C-ski activates cancerassociated fibroblasts to regulate breast cancer cell invasion. Mol Oncol 2013;7:1116-1128.

-33 Wong M, Munro S: Membrane trafficking. The specificity of vesicle traffic to the golgi is encoded in the golgin coiled-coil proteins. Science 2014;346:1256898.

-34 Liu M, Casimiro MC, Wang C, Shirley LA, Jiao X, Katiyar S, Ju X, Li Z, Yu Z, Zhou J, Johnson M, Fortina P, Hyslop T, Windle JJ, Pestell RG: P21cip1 attenuates ras- and c-myc-dependent breast tumor epithelial mesenchymal transition and cancer stem cell-like gene expression in vivo. Proc Natl Acad Sci U S A 2009;106:19035-19039.

-35 Zhang P, Wei Y, Wang L, Debeb BG, Yuan Y, Zhang J, Yuan J, Wang M, Chen D, Sun Y, Woodward WA, Liu Y, Dean DC, Liang H, Hu Y, Ang KK, Hung MC, Chen J, Ma L: Atm-mediated stabilization of zeb1 promotes DNA damage response and radioresistance through chk1. Nat Cell Biol 2014;16:864-875.

-36 Chen WT, Ebelt ND, Stracker TH, Xhemalce B, Van Den Berg CL, Miller KM: Atm regulation of il-8 links oxidative stress to cancer cell migration and invasion. Elife 2015;4:e07270.

37 Paull TT: Mechanisms of atm activation. Annu Rev Biochem 2015;84:711-738.

-38 El Sayegh TY, Arora PD, Fan L, Laschinger CA, Greer PA, McCulloch CA, Kapus A: Phosphorylation of $\mathrm{n}$-cadherin-associated cortactin by fer kinase regulates n-cadherin mobility and intercellular adhesion strength. Mol Biol Cell 2005;16:5514-5527. 


\section{Cellular Physiology and Biochemistry}

Cell Physiol Biochem 2018;51:2972-2988

\begin{tabular}{l|c|c|c|}
\hline DOI: 10.1159/000496048 & ( 2018 The Author(s). Published by S. Karger AG, Basel
\end{tabular}

blished online: 14 December 2018 www.karger.com/cpb

Lang et al.: ATM Phosphorylating Cortactin Contributes to Breast Cancer Migration and Invasion

39 Head JA, Jiang D, Li M, Zorn LJ, Schaefer EM, Parsons JT, Weed SA: Cortactin tyrosine phosphorylation requires rac1 activity and association with the cortical actin cytoskeleton. Mol Biol Cell 2003;14:32163229.

40 Uruno T, Liu J, Zhang P, Fan Y, Egile C, Li R, Mueller SC, Zhan X: Activation of arp2/3 complex-mediated actin polymerization by cortactin. Nat Cell Biol 2001;3:259-266.

41 Weed SA, Karginov AV, Schafer DA, Weaver AM, Kinley AW, Cooper JA, Parsons JT: Cortactin localization to sites of actin assembly in lamellipodia requires interactions with $\mathrm{f}$-actin and the arp $2 / 3$ complex. J Cell Biol 2000;151:29-40.

42 Palmieri D, Valentino T, D’Angelo D, De Martino I, Postiglione I, Pacelli R, Croce CM, Fedele M, Fusco A: Hmga proteins promote atm expression and enhance cancer cell resistance to genotoxic agents. Oncogene 2011;30:3024-3035.

43 Possik PA, Muller J, Gerlach C, Kenski JC, Huang X, Shahrabi A, Krijgsman O, Song JY, Smit MA, Gerritsen B, Lieftink C, Kemper K, Michaut M, Beijersbergen RL, Wessels L, Schumacher TN, Peeper DS: Parallel in vivo and in vitro melanoma rnai dropout screens reveal synthetic lethality between hypoxia and DNA damage response inhibition. Cell Rep 2014;9:1375-1386.

44 Shentu TP, He M, Sun X, Zhang J, Zhang F, Gongol B, Marin TL, Zhang J, Wen L, Wang Y, Geary GG, Zhu Y, Johnson DA, Shyy JY: Amp-activated protein kinase and sirtuin 1 coregulation of cortactin contributes to endothelial function. Arterioscler Thromb Vasc Biol 2016;36:2358-2368. 